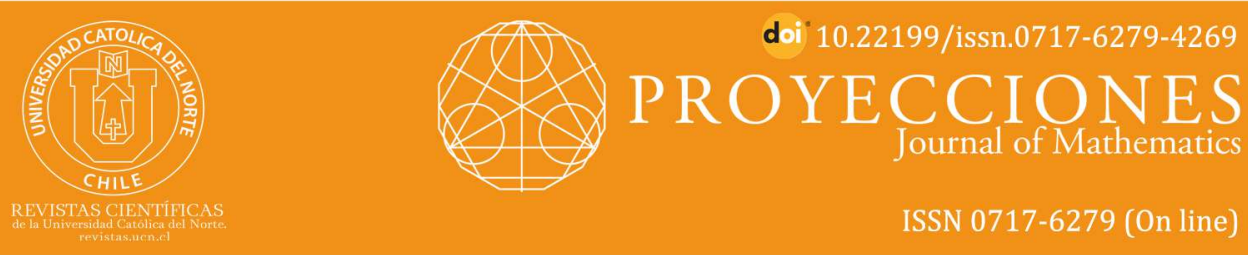

\title{
Some generalized results related to Fibonacci sequence
}

Neeraj Kumar Paul ${ }^{1}$ (i) orcid.org/0000-0003-2804-6564

Helen K. Saikia² ${ }^{2}$ orcid.org/0000-0003-1971-9472

Gauhati University, Dept. of Mathematics, Guwahati, AS, India.

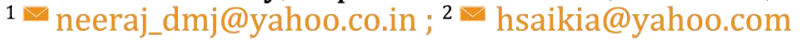

\section{Abstract:}

Cassini's identity states that for the $n^{\text {th }}$ Fibonacci number of the Fibonacci sequence $\left\{F_{n}\right\}, F_{n+1} F_{n-1}-F_{n}^{2}=(-1)^{n}$. We generalize Fibonacci sequence in terms of the number of sequences. Fibonacci sequence is the particular case of generating only one sequence. This generalization is used to generalize Cassini's identity. Moreover we prove few more results which can be seen as generalized form of the results which hold for Fibonacci sequence.

Keywords: Fibonacci sequence; Cassini's identity.

MSC (2020): 11B39.

Cite this article as (IEEE citation style):

N. K. Paul and H. K. Saikia, "Some generalized results related to Fibonacci sequence", Proyecciones (Antofagasta, On line), vol. 40, no. 3, pp. 605-617, 2021, doi: 10.22199/issn.0717-6279-4269

Article copyright: (C) 2021 Neeraj Kumar Paul and Helen K. Saikia. This is an open access article distributed under the terms of the Creative Commons License, which permits unrestricted use and distribution provided the original author and source are credited. 


\section{Introduction}

Fibonacci sequence $\left(F_{k}\right)$ is generated by the recursive formula $F_{k}=F_{k-1}+$ $F_{k-2}$ for $k \geq 3$ with $F_{1}=1, F_{2}=1[2,3]$. That is, each term in the sequence (third term onwards) is the sum of the two terms that immediately precede it. The Fibonacci sequence is the first known recursive sequence in mathematical work. First few terms of the sequence [4] are $1,1,2,3,5,8,13,21, \ldots$. Many generalizations of the sequence and hence its properties are available in the literature.

In [1], we generated $m$ number of sequences following certain recursive rules as follows. When the number of sequence is one, i.e. $m=1$, these rules coincide with those generating Fibonacci numbers and we get the Fibonacci Sequence.

We consider $m$ interconnected sequences

$$
\left(S_{1, k}\right),\left(S_{2, k}\right),\left(S_{3, k}\right), \ldots,\left(S_{m, k}\right)
$$

which can be generated according to the following rule

$$
\begin{array}{ll}
S_{1,1}=S_{2,1}=S_{3,1}=\ldots=S_{m, 1}=1, & S_{1,2}=1, \\
S_{i, k}=S_{i-1, k-1}+S_{i-1, k}, & 1<i \leq m, k \geq 2 \\
S_{1, k}=S_{m, k-1}+S_{m, k-2}, & k \geq 3
\end{array}
$$

\begin{tabular}{|l||r|r|r|r|r|r|}
\hline$k$ & $S_{1, k}$ & $S_{2, k}$ & $S_{3, k}$ & $S_{4, k}$ & $S_{5, k}$ & $S_{6, k}$ \\
\hline \hline 1 & 1 & 1 & 1 & 1 & 1 & 1 \\
\hline 2 & 1 & 2 & 3 & 4 & 5 & 6 \\
\hline 3 & 7 & 8 & 10 & 13 & 17 & 22 \\
\hline 4 & 28 & 35 & 43 & 53 & 66 & 83 \\
\hline 5 & 105 & 133 & 168 & 211 & 264 & 330 \\
\hline 6 & 413 & 518 & 651 & 819 & 1030 & 1294 \\
\hline 7 & 1624 & 2037 & 2555 & 3206 & 4025 & 5055 \\
\hline 8 & 6349 & 7973 & 10010 & 12565 & 15771 & 19796 \\
\hline$\ldots$ & $\ldots$ & $\ldots$ & $\ldots$ & $\ldots$ & $\ldots$ & $\ldots$ \\
\hline
\end{tabular}

Columns show the terms in six sequences (i.e. for $m=6$ ).

For $m=1$, we identify the only sequence $\left(S_{1, k}\right)$ as the Fibonacci sequence $\left(F_{k}\right)$.

Notation: $S_{p, q}$ denotes the $q^{\text {th }}$ term of the $p^{t h}$ sequence.

With this definition we generalized, in [1], the following results for Fibonacci sequence, along with others. 
- $F_{k}+F_{k+1}=F_{k+2}$

Generalization: Sum of $k^{\text {th }}$ terms in all the sequences and $(k+1)^{\text {th }}$ term in the first sequence equals the $(k+2)^{t h}$ term in the first sequence.

- $\sum_{k=1}^{n} F_{k}=F_{n+2}-1$

Generalization: Sum of first $n$ terms of all the sequences is one less than the $(n+2)^{t h}$ term of the first sequence.

- $\sum_{k=0}^{n-1} F_{2 k+1}=F_{2 n}$

Generalization: Sum of all the terms in odd positions (upto $(2 n+$ $1)^{t h}$ position) in all the sequences equals the $(2 n)^{t h}$ term of the last sequence.

- $\sum_{k=1}^{n} F_{2 k}=F_{2 n+1}-1$

Generalization: Sum of all the terms in even positions (upto $(2 n)^{t h}$ position) in all the sequences is one less than the $(2 n+1)^{t h}$ term of the last sequence.

- $\lim _{k \rightarrow \infty} \frac{S_{i, k+1}}{S_{i, k}}$ is a zero of the polynomial $x^{m+1}-(x+1)^{m}$ for $i=$ $1,2, \ldots, m$, which is the generalization of the fact that the golden ratio is a zero of the polynomial $x^{2}-x-1$.

\section{Recurrence relation and few generalizations}

Theorem 2.1. Each sequence can be generated independently by a recurrence relation given by

$$
S_{i, k}={ }^{m} C_{0} S_{i, k-1}+{ }^{m} C_{1} S_{i, k-2}+{ }^{m} C_{2} S_{i, k-3}+\ldots+{ }^{m} C_{m} S_{i, k-m-1}
$$

with initial terms being for $1 \leq i \leq m$ and $-(m+1) \leq k \leq-1$

$$
S_{i, k}=(-1)^{i+k+m+1}\left({ }^{-k-1} C_{m-i}\right)
$$

Proof. We restate the rules for generating the sequences.

We set 


$$
\begin{array}{ll}
S_{1,1}=S_{2,1}=S_{3,1}=\ldots=S_{m, 1}=1, & S_{1,2}=1, \\
S_{i, k}=S_{i-1, k-1}+S_{i-1, k}, & 1<i \leq m, k \geq 2 \\
S_{1, k}=S_{m, k-1}+S_{m, k-2}, & k \geq 3
\end{array}
$$

Extending the sequences backward, we can easily see that for $1 \leq i \leq m$,

$$
S_{i, 0}=0,
$$

and for $-(m+1) \leq k \leq-1$

$$
S_{i, k}=(-1)^{i+k+m+1}\left({ }^{-k-1} C_{m-i}\right)
$$

Clearly, $S_{i, k}=0$ for $i-m \leq k \leq-1$ and $S_{m,-1}=1$.

We now make a claim for the recurrence relation. A term in a sequence can be obtained using the following recurring relation involving the previously obtained $(m+1)$ terms of the same sequence.

$$
S_{i, k}={ }^{m} C_{0} S_{i, k-1}+{ }^{m} C_{1} S_{i, k-2}+{ }^{m} C_{2} S_{i, k-3}+\ldots+{ }^{m} C_{m} S_{i, k-m-1}
$$

with initial terms being $S_{i, k}$, as defined in (2.1), where $1 \leq i \leq m$ and $-(m+1) \leq k \leq-1$.

Now,

$$
\begin{aligned}
S_{i, 0} & ={ }^{m} C_{0} S_{i,-1}+{ }^{m} C_{1} S_{i,-2}+{ }^{m} C_{2} S_{i,-3}+\ldots+{ }^{m} C_{m} S_{i,-m-1} \\
& =0
\end{aligned}
$$

Thus $S_{i, k}=S_{i-1, k-1}+S_{i-1, k}$, for $1<i \leq m,-m \leq k \leq 0$

and $S_{1, k}=S_{m, k-1}+S_{m, k-2}$, for $-m+1 \leq k \leq 0$

are satisfied.

These are also satisfied for $k=1$ as

$$
\begin{aligned}
S_{i, 1} & ={ }^{m} C_{0} S_{i, 0}+{ }^{m} C_{1} S_{i,-1}+{ }^{m} C_{2} S_{i,-2}+\ldots+{ }^{m} C_{m} S_{i,-m} \\
& =1
\end{aligned}
$$

We now establish the recurrence relation (2.2) using induction on $k$. Suppose the definition of the sequences are satisfied for $k \leq l$. Then 


$$
\begin{aligned}
S_{m, l-1}+S_{m, l}= & { }^{m} C_{0} S_{m, l-2}+{ }^{m} C_{1} S_{m, l-3}+{ }^{m} C_{2} S_{m, l-4}+ \\
& \ldots+{ }^{m} C_{m} S_{m, l-m-2}+ \\
& { }^{m} C_{0} S_{m, l-1}+{ }^{m} C_{1} S_{m, l-2}+{ }^{m} C_{2} S_{m, l-3}+ \\
= & \left.{ }^{m}{ }^{m}{ }^{m} C_{m} S_{m, l-m-1} S_{m, l-2}+S_{m, l-1}\right)+{ }^{m} C_{1}\left(S_{m, l-3}+S_{m, l-2}\right) \\
& +{ }^{m} C_{2}\left(S_{m, l-4}+S_{m, l-3}\right)+\ldots \\
& +{ }^{m} C_{m}\left(S_{m, l-m-2}+S_{m, l-m-1}\right) \\
= & { }^{m} C_{0} S_{1, l}+{ }^{m} C_{1} S_{1, l-1}+{ }^{m} C_{2} S_{1, l-2}+ \\
& \ldots+{ }^{m} C_{m} S_{1, l-m} \\
= & S_{1, l+1}
\end{aligned}
$$

and for $1<i \leq m$

$$
\begin{aligned}
S_{i-1, l-1}+S_{i-1, l}= & { }^{m} C_{0} S_{i-1, l-2}+{ }^{m} C_{1} S_{i-1, l-3}+{ }^{m} C_{2} S_{i-1, l-4}+ \\
& \ldots+{ }^{m} C_{m} S_{i-1, l-m-2} \\
& +{ }^{m} C_{0} S_{i-1, l-1}+{ }^{m} C_{1} S_{i-1, l-2}+{ }^{m} C_{2} S_{i-1, l-3}+ \\
& \ldots+{ }^{m} C_{m} S_{i-1, l-m-1} \\
= & S_{i, l}
\end{aligned}
$$

Hence the claim.

Theorem 2.2. For each of the $m$ sequences, gcd of $(m+1)$ consecutive terms is 1. This is generalization of the fact that pair of consecutive Fibonacci numbers are relatively prime.

Proof. We first write $m+1$ consecutive terms of a particular sequence as below.

$$
\begin{aligned}
& S_{i, k}={ }^{m} C_{0} S_{i, k-1}+{ }^{m} C_{1} S_{i, k-2}+\ldots+{ }^{m} C_{m} S_{i, k-m-1} \\
& S_{i, k+1}={ }^{m} C_{0} S_{i, k}+{ }^{m} C_{1} S_{i, k-1}+\ldots+{ }^{m} C_{m} S_{i, k-m} \\
& S_{i, k+2}={ }^{m} C_{0} S_{i, k+1}+{ }^{m} C_{1} S_{i, k}+\ldots+{ }^{m} C_{m} S_{i, k-m+1} \\
& \begin{array}{lllll}
\text {. } & \ldots & \ldots & \ldots & \ldots
\end{array} \\
& \begin{array}{lllll}
\ldots & \ldots & \ldots & \ldots & \ldots
\end{array} \\
& S_{i, k+m-1}={ }^{m} C_{0} S_{i, k+m-2}+{ }^{m} C_{1} S_{i, k+m-3}+\ldots+{ }^{m} C_{m} S_{i, k-2} \\
& S_{i, k+m}={ }^{m} C_{0} S_{i, k+m-1}+{ }^{m} C_{1} S_{i, k+m-2}+\ldots+{ }^{m} C_{m} S_{i, k-1}
\end{aligned}
$$

Suppose $g$ divides all the above terms. Then from the last expression, we can write

${ }^{m} C_{m} S_{i, k-1}=S_{i, k+m}-{ }^{m} C_{0} S_{i, k+m-1}-{ }^{m} C_{1} S_{i, k+m-2}-\ldots-{ }^{m} C_{m-1} S_{i, k}$ which implies that $g$ divides $S_{i, k-1}$.

Now considering the fact that $g$ divides the $m+1$ consecutive terms $S_{i, k-1}, S_{i, k}, S_{i, k+1}, S_{i, k+2}, \ldots, S_{i, k+m-1}$, we proceed as above to get $g$ 
divides $S_{i, k-2}$. Continuing in similar fashion, we obtain that $g$ divides $S_{i, 1}$. This implies $g=1$.

Theorem 2.3. Generalization of Cassini's Identity $F_{k+1} F_{k-1}-F_{k}^{2}=(-1)^{k}$

For $i^{\text {th }}$ sequence,

$$
\begin{aligned}
& \left|\begin{array}{lllll}
S_{i, k+1} & S_{i, k} & S_{i, k-1} & \ldots & S_{i, k-(m-1)} \\
S_{i, k} & S_{i, k-1} & S_{i, k-2} & \ldots & S_{i, k-m} \\
S_{i, k-1} & S_{i, k-2} & S_{i, k-3} & \ldots & S_{i, k-(m+1)} \\
\ldots & \ldots & \ldots & \ldots & \ldots \\
S_{i, k-(m-2)} & S_{i, k-(m-1)} & S_{i, k-m} & \ldots & S_{i, k-(2 m-2)} \\
S_{i, k-(m-1)} & S_{i, k-m} & S_{i, k-(m+1)} & \ldots & S_{i, k-(2 m-1)}
\end{array}\right| \\
& =(-1)^{m(i-1)}\left\{\begin{array}{l}
(-1)^{\frac{m}{2}} \text { when } m \text { is even, } \\
(-1)^{\frac{m-1}{2}} \text { when } m \text { is odd, and } k \text { is even, } \\
(-1)^{\frac{m+1}{2}} \text { when both } m \text { and } k \text { are odd. }
\end{array}\right.
\end{aligned}
$$

Proof. We write

$$
\begin{aligned}
& S_{i, k+m}={ }^{m} C_{0} S_{i, k+m-1}+{ }^{m} C_{1} S_{i, k+m-2}+\ldots+{ }^{m} C_{m-1} S_{i, k}+{ }^{m} C_{m} S_{i, k-1} \\
& S_{i, k+m-1}=1 S_{i, k+m-1}+0 S_{i, k+m-2}+\ldots+\quad 0 S_{i, k}+0 S_{i, k-1} \\
& S_{i, k+m-2}=0 S_{i, k+m-1}+1 S_{i, k+m-2}+\ldots+\quad 0 S_{i, k}+\quad 0 S_{i, k-1} \\
& \begin{array}{lllll}
\ldots & \ldots & \ldots & \ldots & \ldots
\end{array} \\
& \begin{array}{cccccc}
\cdots & \cdots & \cdots & \cdots & \cdots & \cdots \\
S_{i, k} & = & 0 S_{i, k+m-1}+ & 0 S_{i, k+m-2}+\ldots+ & 1 S_{i, k}+ & 0 S_{i, k-1}
\end{array}
\end{aligned}
$$

In matrix form,

$$
\left[\begin{array}{l}
S_{i, k+m} \\
S_{i, k+m-1} \\
S_{i, k+m-2} \\
S_{i, k+m-3} \\
\cdots \\
S_{i, k+1} \\
S_{i, k}
\end{array}\right]=\left[\begin{array}{cccccc}
{ }^{m} C_{0} & { }^{m} C_{1} & { }^{m} C_{2} & \ldots & { }^{m} C_{m-1} & { }^{m} C_{m} \\
1 & 0 & 0 & \ldots & 0 & 0 \\
0 & 1 & 0 & \ldots & 0 & 0 \\
0 & 0 & 1 & \ldots & 0 & 0 \\
\ldots & \ldots & \ldots & \ldots & \ldots & \ldots \\
0 & 0 & 0 & \ldots & 0 & 0 \\
0 & 0 & 0 & \ldots & 1 & 0
\end{array}\right]\left[\begin{array}{l}
S_{i, k+m-1} \\
S_{i, k+m-2} \\
S_{i, k+m-3} \\
S_{i, k+m-4} \\
\ldots \\
S_{i, k} \\
S_{i, k-1}
\end{array}\right]
$$


We define

$$
A=\left[\begin{array}{cccccc}
{ }^{m} C_{0} & { }^{m} C_{1} & { }^{m} C_{2} & \ldots & { }^{m} C_{m-1} & { }^{m} C_{m} \\
1 & 0 & 0 & \ldots & 0 & 0 \\
0 & 1 & 0 & \cdots & 0 & 0 \\
0 & 0 & 1 & \ldots & 0 & 0 \\
\cdots & \ldots & \ldots & \cdots & \cdots & \ldots \\
0 & 0 & 0 & \ldots & 0 & 0 \\
0 & 0 & 0 & \cdots & 1 & 0
\end{array}\right]
$$

Then $A^{k}$ equals

$$
\left[\begin{array}{lllll}
\sum_{j=0}^{m}{ }^{m} C_{j} S_{1, k-j} & \sum_{j=1}^{m}{ }^{m} C_{j} S_{1, k+1-j} & \ldots & \sum_{j=m-1}^{m}{ }^{m} C_{j} S_{1, k+(m-1)-j} & S_{1, k} \\
\sum_{j=0}^{m}{ }^{m} C_{j} S_{1, k-1-j} & \sum_{j=1}^{m}{ }^{m} C_{j} S_{1, k-j} & \ldots & \sum_{j=m-1}^{m}{ }^{m} C_{j} S_{1,(k-1)+(m-1)-j} & S_{1, k-1} \\
\sum_{j=0}^{m}{ }^{m} C_{j} S_{1, k-2-j} & \sum_{j=1}^{m}{ }^{m} C_{j} S_{1, k-1-j} & \ldots & \sum_{j=m-1}^{m}{ }^{m} C_{j} S_{1,(k-2)+(m-1)-j} & S_{1, k-2} \\
\ldots & \ldots & \ldots & \ldots & \ldots \\
\sum_{j=0}^{m}{ }^{m} C_{j} S_{1, k-(m-1)-j} & \sum_{j=1}^{m}{ }^{m} C_{j} S_{1, k-(m-2)-j} & \ldots & \sum_{j=m-1}^{m}{ }^{m} C_{j} S_{1, k-j} & S_{1, k-(m-1)} \\
\sum_{j=0}^{m}{ }^{m} C_{j} S_{1, k-m-j} & \sum_{j=1}^{m}{ }^{m} C_{j} S_{1, k-(m-1)-j} & \ldots & \sum_{j=m-1}^{m}{ }^{m} C_{j} S_{1, k-m+(m-1)-j} & S_{1, k-m}
\end{array}\right]
$$

$(p, q)$ th entry in the above $(m+1) \times(m+1)$ matrix is $\sum_{j=q-1}^{m}{ }^{m} C_{j} S_{1, k-(p-q)-j}$. 
or, $A^{k}$ equals

$\left[\begin{array}{lllll}S_{1, k+1} & \sum_{j=1}^{m}{ }^{m} C_{j} S_{1, k+1-j} & \ldots & \sum_{j=m-1}^{m}{ }^{m} C_{j} S_{1, k+(m-1)-j} & S_{1, k} \\ S_{1, k} & \sum_{j=1}^{m}{ }^{m} C_{j} S_{1, k-j} & \ldots & \sum_{j=m-1}^{m}{ }^{m} C_{j} S_{1,(k-1)+(m-1)-j} & S_{1, k-1} \\ S_{1, k-1} & \sum_{j=1}^{m}{ }^{m} C_{j} S_{1, k-1-j} & \ldots & \sum_{j=m-1}^{m}{ }^{m} C_{j} S_{1,(k-2)+(m-1)-j} & S_{1, k-2} \\ \ldots & \cdots & \ldots & \cdots & \ldots \\ S_{1, k-(m-2)} & \sum_{j=1}^{m}{ }^{m} C_{j} S_{1, k-(m-2)-j} & \ldots & \sum_{j=m-1}^{m}{ }^{m} C_{j} S_{1, k-j} & S_{1, k-(m-1)} \\ S_{1, k-(m-1)} & \sum_{j=1}^{m}{ }^{m} C_{j} S_{1, k-(m-1)-j} & \ldots & \sum_{j=m-1}^{m}{ }^{m} C_{j} S_{1, k-m+(m-1)-j} & S_{1, k-m}\end{array}\right]$

so that $\left|A^{k}\right|$ equals

\begin{tabular}{|lllll}
$S_{1, k+1}$ & $\sum_{j=1}^{m}{ }^{m} C_{j} S_{1, k+1-j}$ & $\ldots$ & $\sum_{j=m-1}^{m}{ }^{m} C_{j} S_{1, k+(m-1)-j}$ & $S_{1, k}$ \\
$S_{1, k}$ & $\sum_{j=1}^{m}{ }^{m} C_{j} S_{1, k-j}$ & $\ldots$ & $\sum_{j=m-1}^{m}{ }^{m} C_{j} S_{1,(k-1)+(m-1)-j}$ & $S_{1, k-1}$ \\
$S_{1, k-1}$ & $\sum_{j=1}^{m}{ }^{m} C_{j} S_{1, k-1-j}$ & $\ldots$ & $\sum_{j=m-1}^{m}{ }^{m} C_{j} S_{1,(k-2)+(m-1)-j}$ & $S_{1, k-2}$ \\
$\ldots$ & $\ldots$ & $\ldots$ & $\ldots$ \\
$S_{1, k-(m-2)}$ & $\sum_{j=1}^{m}{ }^{m} C_{j} S_{1, k-(m-2)-j}$ & $\ldots$ & $\sum_{j=m-1}^{m}{ }^{m} C_{j} S_{1, k-j}$ & $S_{1, k-(m-1)}$ \\
$S_{1, k-(m-1)}$ & $\sum_{j=1}^{m}{ }^{m} C_{j} S_{1, k-(m-1)-j}$ & $\ldots$ & $\sum_{j=m-1}^{m}{ }^{m} C_{j} S_{1, k-m+(m-1)-j}$ & $S_{1, k-m}$
\end{tabular} \mid


Applying column operations, we get

$$
\begin{array}{|llllll|}
S_{1, k+1} & S_{1, k-(m-1)} & S_{1, k-(m-2)} & \ldots & S_{1, k-1} & S_{1, k} \\
S_{1, k} & S_{1, k-m} & S_{1, k-(m-1)} & \ldots & S_{1, k-2} & S_{1, k-1} \\
S_{1, k-1} & S_{1, k-(m+1)} & S_{1, k-m} & \ldots & S_{1, k-3} & S_{1, k-2} \\
\ldots & \ldots & \ldots & \ldots & \ldots & \ldots \\
S_{1, k-(m-2)} & S_{1, k-(2 m-2)} & S_{1, k-(2 m-3)} & \ldots & S_{1, k-m} & S_{1, k-(m-1)} \\
S_{1, k-(m-1)} & S_{1, k-(2 m-1)} & S_{1, k-(2 m-2)} & \ldots & S_{1, k-(m+1)} & S_{1, k-m} \\
& \\
& & &
\end{array}
$$

Rearranging the columns, we obtain

$$
\begin{aligned}
& \left|\begin{array}{lllll}
S_{1, k+1} & S_{1, k} & S_{1, k-1} & \ldots & S_{1, k-(m-1)} \\
S_{1, k} & S_{1, k-1} & S_{1, k-2} & \ldots & S_{1, k-m} \\
S_{1, k-1} & S_{1, k-2} & S_{1, k-3} & \ldots & S_{1, k-(m+1)} \\
\ldots & \ldots & \ldots & \ldots & \ldots \\
S_{1, k-(m-2)} & S_{1, k-(m-1)} & S_{1, k-m} & \ldots & S_{1, k-(2 m-2)} \\
S_{1, k-(m-1)} & S_{1, k-m} & S_{1, k-(m+1)} & \ldots & S_{1, k-(2 m-1)}
\end{array}\right| \\
& \quad=\left\{\begin{array}{lll}
(-1)^{\frac{m}{2}}(-1)^{m k} & \text { when } m \text { is even, } \\
(-1)^{\frac{m-1}{2}}(-1)^{m k} & \text { when } m \text { is odd. }
\end{array}\right.
\end{aligned}
$$

Thus,

$$
\begin{aligned}
& \left|\begin{array}{lllll}
S_{1, k+1} & S_{1, k} & S_{1, k-1} & \ldots & S_{1, k-(m-1)} \\
S_{1, k} & S_{1, k-1} & S_{1, k-2} & \ldots & S_{1, k-m} \\
S_{1, k-1} & S_{1, k-2} & S_{1, k-3} & \ldots & S_{1, k-(m+1)} \\
\ldots & \ldots & \ldots & \ldots & \ldots \\
S_{1, k-(m-2)} & S_{1, k-(m-1)} & S_{1, k-m} & \ldots & S_{1, k-(2 m-2)} \\
S_{1, k-(m-1)} & S_{1, k-m} & S_{1, k-(m+1)} & \ldots & S_{1, k-(2 m-1)}
\end{array}\right| \\
& \quad=\left\{\begin{array}{lll}
(-1)^{\frac{m}{2}} & \text { when } m \text { is even, } \\
(-1)^{\frac{m-1}{2}} & \text { when } m \text { is odd, } k \text { is even, } \\
(-1)^{\frac{m+1}{2}} & \text { when } m \text { and } k \text { are odd. }
\end{array}\right.
\end{aligned}
$$




\section{Suppose}

$$
\left|\begin{array}{llllll}
S_{i, k+1} & S_{i, k} & S_{i, k-1} & \ldots & S_{i, k-(m-2)} & S_{i, k-(m-1)} \\
S_{i, k} & S_{i, k-1} & S_{i, k-2} & \ldots & S_{i, k-(m-1)} & S_{i, k-m} \\
S_{i, k-1} & S_{i, k-2} & S_{i, k-3} & \ldots & S_{i, k-m} & S_{i, k-(m+1)} \\
\ldots & \ldots & \ldots & \ldots & \ldots & \ldots \\
S_{i, k-(m-2)} & S_{i, k-(m-1)} & S_{i, k-m} & \ldots & S_{i, k-(2 m-3)} & S_{i, k-(2 m-2)} \\
S_{i, k-(m-1)} & S_{i, k-m} & S_{i, k-(m+1)} & \ldots & S_{i, k-(2 m-2)} & S_{i, k-(2 m-1)}
\end{array}\right|=\Delta
$$

By $C_{j} \rightarrow C_{j}+C_{j+1}$, for $j=1,2, \ldots, m$

$$
\left|\begin{array}{llllll}
S_{i+1, k+1} & S_{i+1, k} & S_{i+1, k-1} & \ldots & S_{i+1, k-(m-2)} & S_{i, k-(m-1)} \\
S_{i+1, k} & S_{i+1, k-1} & S_{i+1, k-2} & \ldots & S_{i+1, k-(m-1)} & S_{i, k-m} \\
S_{i+1, k-1} & S_{i+1, k-2} & S_{i+1, k-3} & \ldots & S_{i+1, k-m} & S_{i, k-(m+1)} \\
\ldots & \ldots & \ldots & \ldots & \ldots & \ldots \\
S_{i+1, k-(m-2)} & S_{i+1, k-(m-1)} & S_{i+1, k-m} & \ldots & S_{i+1, k-(2 m-3)} & S_{i, k-(2 m-2)} \\
S_{i+1, k-(m-1)} & S_{i+1, k-m} & S_{i+1, k-(m+1)} & \ldots & S_{i+1, k-(2 m-2)} & S_{i, k-(2 m-1)}
\end{array}\right|=\Delta
$$

Also

$$
\begin{aligned}
S_{i+1, k}= & S_{i+1, k+m} \\
& -\left(1+{ }^{m} C_{0}\right) S_{i+1, k+m-1} \\
& +\left(1+{ }^{m} C_{0}-{ }^{m} C_{1}\right) S_{i+1, k+m-2} \\
& -\left(1+{ }^{m} C_{0}-{ }^{m} C_{1}+{ }^{m} C_{2}\right) S_{i+1, k+m-3} \\
& +\ldots \\
& +(-1)^{m-1}\left(1+{ }^{m} C_{0}-{ }^{m} C_{1}+{ }^{m} C_{2}-\ldots+{ }^{m} C_{m-2}\right) S_{i+1, k+1} \\
& +(-1)^{m} S_{i, k}
\end{aligned}
$$

Applying column operation on the last column as per the above formula, we get

$$
\begin{aligned}
& \left|\begin{array}{llllll}
S_{i+1, k+1} & S_{i+1, k} & S_{i+1, k-1} & \ldots & S_{i+1, k-(m-2)} & S_{i+1, k-(m-1)} \\
S_{i+1, k} & S_{i+1, k-1} & S_{i+1, k-2} & \ldots & S_{i+1, k-(m-1)} & S_{i+1, k-m} \\
S_{i+1, k-1} & S_{i+1, k-2} & S_{i+1, k-3} & \ldots & S_{i+1, k-m} & S_{i+1, k-(m+1)} \\
\ldots & \ldots & \ldots & \ldots & \ldots & \ldots \\
S_{i+1, k-(m-2)} & S_{i+1, k-(m-1)} & S_{i+1, k-m} & \ldots & S_{i+1, k-(2 m-3)} & S_{i+1, k-(2 m-2)} \\
S_{i+1, k-(m-1)} & S_{i+1, k-m} & S_{i+1, k-(m+1)} & \ldots & S_{i+1, k-(2 m-2)} & S_{i+1, k-(2 m-1)}
\end{array}\right| \\
& \quad \begin{array}{llll} 
\\
\quad=(-1)^{m} \Delta
\end{array}
\end{aligned}
$$


Thus we generalize Cassini's identity as

$$
\begin{aligned}
& \left|\begin{array}{lllll}
S_{i, k+1} & S_{i, k} & S_{i, k-1} & \ldots & S_{i, k-(m-1)} \\
S_{i, k} & S_{i, k-1} & S_{i, k-2} & \ldots & S_{i, k-m} \\
S_{i, k-1} & S_{i, k-2} & S_{i, k-3} & \ldots & S_{i, k-(m+1)} \\
\ldots & \ldots & \ldots & \ldots & \ldots \\
S_{i, k-(m-2)} & S_{i, k-(m-1)} & S_{i, k-m} & \ldots & S_{i, k-(2 m-2)} \\
S_{i, k-(m-1)} & S_{i, k-m} & S_{i, k-(m+1)} & \ldots & S_{i, k-(2 m-1)}
\end{array}\right| \\
& \quad=(-1)^{m(i-1)} \begin{cases}(-1)^{\frac{m}{2}} & \text { when } m \text { is even, } \\
(-1)^{\frac{m-1}{2}} & \text { when } m \text { is odd, } \\
& \text { and } k \text { is even, } \\
(-1)^{\frac{m+1}{2}} & \text { when both } \\
& m \text { and } k \text { are odd. }\end{cases}
\end{aligned}
$$

For $m=1$, this reduces to a $2 \times 2$ determinant with $i=1$.

That is

$$
\left|\begin{array}{ll}
S_{1, k+1} & S_{1, k} \\
S_{1, k} & S_{1, k-1}
\end{array}\right|=\left\{\begin{aligned}
1 & \text { when } k \text { is even, } \\
-1 & \text { when } k \text { is odd. }
\end{aligned}\right.
$$

which is the Cassini's identity $F_{k+1} F_{k-1}-F_{k}^{2}=(-1)^{k}$ for Fibonacci numbers. Note that for $m=1, S_{1, k}=F_{k}$.

Theorem 2.4. Generalization of $F_{p+q}=F_{p+1} F_{q}+F_{p} F_{q-1}$

$$
\begin{aligned}
S_{1, p+q}= & \left(\sum_{j=0}^{m}{ }^{m} C_{j} S_{1, p-j}\right) S_{1, q}+\left(\sum_{j=1}^{m}{ }^{m} C_{j} S_{1, p-j+1}\right) S_{1, q-1} \\
& +\left(\sum_{j=2}^{m}{ }^{m} C_{j} S_{1, p-j+2}\right) S_{1, q-2} \\
& +\ldots+\left(\sum_{j=m-1}^{m}{ }^{m} C_{j} S_{1, p-j+(m-1)}\right) S_{1, q-(m-1)}+S_{1, p} S_{1, q-m}
\end{aligned}
$$


Proof. $\quad A^{p} A^{q}=A^{p+q}$ gives

$$
\begin{aligned}
S_{1, p+q+1}= & \left(\sum_{j=0}^{m}{ }^{m} C_{j} S_{1, p-j}\right)\left(\sum_{j=0}^{m}{ }^{m} C_{j} S_{1, q-j}\right) \\
& +\left(\sum_{j=1}^{m}{ }^{m} C_{j} S_{1, p-j+1}\right)\left(\sum_{j=0}^{m}{ }^{m} C_{j} S_{1, q-j-1}\right) \\
& +\left(\sum_{j=2}^{m}{ }^{m} C_{j} S_{1, p-j+2}\right)\left(\sum_{j=0}^{m}{ }^{m} C_{j} S_{1, q-j-2}\right) \\
& +\ldots+\left(\sum_{j=m-1}^{m}{ }^{m} C_{j} S_{1, p-j+(m-1)}\right)\left(\sum_{j=0}^{m}{ }^{m} C_{j} S_{1, q-j-(m-1)}\right) \\
& +S_{1, p}\left(\sum_{j=0}^{m}{ }^{m} C_{j} S_{1, q-j-m}\right)
\end{aligned}
$$

Note: $S_{1, q}=\sum_{j=0}^{m}{ }^{m} C_{j} S_{1,(q-1)-j}$.

Therefore

$$
\begin{aligned}
S_{1, p+q+1}= & \left(\sum_{j=0}^{m}{ }^{m} C_{j} S_{1, p-j}\right) S_{1, q+1}+\left(\sum_{j=1}^{m}{ }^{m} C_{j} S_{1, p-j+1}\right) S_{1, q} \\
& +\left(\sum_{j=2}^{m}{ }^{m} C_{j} S_{1, p-j+2}\right) S_{1, q-1} \\
& +\ldots+\left(\sum_{j=m-1}^{m}{ }^{m} C_{j} S_{1, p-j+(m-1)}\right) S_{1, q-(m-2)}+S_{1, p} S_{1, q-(m-1)}
\end{aligned}
$$

Writing $q-1$ for $q$, we get

$$
\begin{aligned}
S_{1, p+q}= & \left(\sum_{j=0}^{m}{ }^{m} C_{j} S_{1, p-j}\right) S_{1, q}+\left(\sum_{j=1}^{m}{ }^{m} C_{j} S_{1, p-j+1}\right) S_{1, q-1} \\
& +\left(\sum_{j=2}^{m}{ }^{m} C_{j} S_{1, p-j+2}\right) S_{1, q-2} \\
& +\ldots+\left(\sum_{j=m-1}^{m}{ }^{m} C_{j} S_{1, p-j+(m-1)}\right) S_{1, q-(m-1)}+S_{1, p} S_{1, q-m}
\end{aligned}
$$




\section{Conclusion}

The generalization of Fibonacci sequence as discussed in this paper generalizes Cassini's identity in matrix form. Binet's Formula, Catalan's identities etc. can be explored to be generalized accordingly.

\section{Acknowledgement}

The first author thanks University Grants Commission of India for its financial support to the work.

\section{References}

[1] N. K. Paul and H. K. Saikia, "A generalization of Fibonacci sequence", Proyecciones (Antofagasta), vol. 39, no. 6, pp. 1393-1405, 2020, doi: 10.22199/ issn.0717-6279-2020-06-0085

[2] D. M. Burton, Elementary number theory, 7th ed. Boston, MA: McGrawHill, 2011.

[3] G. H. Hardy and E. M. Wright, An introduction to the theory of numbers, 6th ed. Oxford: Oxford University Press, 2008.

[4] N. J. A. Sloane, "A000045:Fibonacci numbers: $F(n)=F(n-1) F(n-2)$ with $F(0)=0$ and $F(1)=1$.", The on-line encyclopedia of integer sequences. The OEIS Foundation, 2011 [Online]. Available: https://oeis.org/ A000045 